\title{
Clinical management of uveal melanoma: a comprehensive review with a treatment algorithm
}

\author{
Mutlay Sayan', Swati Mamidanna', Damla Oncel², Imraan Jan', Irina Vergalasova', Joseph Weiner ${ }^{1}$, Nisha Ohri' \\ Banu Acikalin", Anupama Chundury ${ }^{1}$ \\ ${ }^{1}$ Department of Radiation Oncology, Rutgers Cancer Institute of New Jersey, New Brunswick, NJ, USA \\ ${ }^{2}$ Department of Biochemistry, University of California, Los Angeles, CA, USA \\ ${ }^{3}$ Department of Ophthalmology, Istanbul Fatih Sultan Mehmet Education and Research Hospital, Istanbul, Turkey
}

Received: May 27, 2020

Revised: June 17, 2020

Accepted: June 19, 2020

\section{Correspondence:}

Mutlay Sayan

Department of Radiation Oncology,

Rutgers Cancer Institute of New

Jersey, 195 Little Albany Street, New

Brunswick, NJ 08901, USA.

Tel: +1-732-235-3939

E-mail: ms2641@cinj.rutgers.edu

ORCID:

https://orcid.org/0000-0002-0101-0951

\begin{abstract}
Uveal melanoma (UM), the most frequently occurring non-cutaneous melanoma and most common primary intraocular malignancy in adults, arises from the melanocytes of the choroid in approximately $95 \%$ of cases. Prompt diagnosis and treatment is vital as primary tumor size is one of the key factors associated with survival. Despite recent advances in management, more than half of the patients develop metastatic disease which portends poor survival. Currently, treatment options for UM include local resection, enucleation, plaque brachytherapy, and/or particle beam radiotherapy (RT). Enucleation was initially the standard of care in the management of UM, but a shift towards eye-preserving therapeutic choices such as RT and local resection has been noted in recent decades. Plaque brachytherapy, a form of localized RT, is the most popular option and is now the preferred treatment modality for a majority of UM cases. In this review we discuss the etiopathogenesis, clinical presentation and diagnosis of UM and place a special emphasis on its therapeutic options. Furthermore, we review the current literature on UM management and propose a functional treatment algorithm for non-metastatic disease.
\end{abstract}

Keywords: Uveal melanoma, Surgery, Radiotherapy, Brachytherapy

\section{Introduction}

Melanoma is a malignancy that originates from the neoplastic proliferation of melanin-producing cells known as melanocytes, which can be primarily found in the skin, ocular region and mucous membranes. Uveal melanoma (UM) is the most frequently occurring non-cutaneous melanoma and is the most common primary intraocular malignancy in adults [1]. The uveal tract, a layer underlying the sclera of the eye, includes the iris, ciliary body and choroid. Around $95 \%$ of uveal melanomas arise from the choroidal melanocytes.

\section{Epidemiology}

The worldwide incidence of UM is estimated to be close to 4 to 5 cases per million per year and varies with gender, race and geographical location. Even though most studies reveal no apparent gender preponderance, some European studies have seen a slightly increased incidence in males [2]. Caucasians are most commonly affected ethic group, accounting for 98\% of UM cases. An increase in incidence of UM with latitude has also been observed with a decreasing gradient of cases from Northern to Southern Europe being reported. Furthermore, lower incidences of UM have also been noted in Asian and African nations [3].

\section{Etiopathogenesis}

As with many other types of malignancies, the precise causative elements for this rare cancer have yet to be clearly established. Both genetic and environmental risk factors have been implicated

Copyright (C) 2020 The Korean Society for Radiation Oncology

This is an Open Access article distributed under the terms of the Creative Commons Attribution Non-Commercial License (http://creativecommons.org/licenses/by-nc/4.0/) which permits unrestricted non-commercial use, distribution, and reproduction in any medium, provided the original work is properly cited. 
in the etiology of developing UM. Genetic factors previously associated include fair skin, inability to tan and light eye color [4]. A positive family history of UM, increased frequency of oculodermal melanocytosis, BAP1 mutations and dysplastic nevi have also been linked to a higher incidence of UM [5].

Although sunlight exposure is an independent risk factor in the development of cutaneous melanoma, epidemiological attempts to analyze the association between exposure to ultraviolet (UV) light and UM have led to contradicting results. Some studies suggest that UV light exposure is a synergistic risk factor for developing UM in individuals with light iris color [6]. Mutation mechanisms with GNAQ and GNA11 signatures noted in illuminated areas of the uvea have also suggested that sunlight exposure may be an independent risk [7]. However, as previously stated, other attempts to associate intermittent and chronic UV light exposure with development of UM have led to inconsistent results [8]. The ability of the cornea and lens to filter a major portion of UV radiation in sunlight before it reaches the uveal tissue has been suggested as a possible explanation for this unclear association [9]. Finally, occupational hazards, such as welding, have also been associated as an etiologic source for developing UM as it usually involves additional chemical exposure along with both infrared and visible radiation.

\section{Clinical features}

Nearly half of the patients at the time of diagnosis are asymptomatic and UM is only identified after routine eye examination. Symptomatic patients present with ophthalmological features such as floaters, photopsia, visual field defects, metamorphopsia, visible tumor, and/or painless loss of vision [10]. Iris melanomas are diagnosed almost 15-20 years earlier than choroidal or ciliary body melanomas, primarily owing to early iris color changes and distortion of the pupil.

\section{Diagnosis}

There are significant differential diagnoses of UM that include choroidal nevus, retinal pigment epithelium hypertrophy and disciform degeneration, making it is integral to obtain an accurate diagnosis as soon as possible. A fundoscopic examination is the first step in obtaining this goal. Presence of orange pigment, subretinal fluid, and documentation of tumor growth all aid in obtaining an appropriate diagnosis [11]. Further evaluation is needed for additional tumor characterization with procedures such as optical coherence tomography ultrasound and/or fluorescein angiography. Ultrasound features of UM include an intrinsic acoustic quiet zone and decreased internal reflectivity of the tumor. Visually, the tumor is often described as circumscribed mushroom or dome-shaped mass after rupture of the Bruch's membrane of the retina [12]. Fluores- cein angiography helps to evaluate the vascular supply of the tumor. A biopsy is not required for diagnosis, but is often performed for prognostic purposes. An understanding of the molecular structure of the tumor helps in risk stratification and determining initial treatment paradigms.

\section{Prognosis}

Despite advances in UM therapies, almost half of the patients are ultimately at risk for developing metastatic disease, primarily due to the inability to identify a unique feature of UM which is early micro-metastases [13]. Owing to this frequent subsequent presentation, patients diagnosed with UM require periodic surveillance with physical examination, blood tests and radiographic imaging such as $\mathrm{CT}, \mathrm{MRI}$, abdominal ultrasound or PET/CT. The most common initial distant metastatic sites include the liver followed by the lung, skin and bone. Ciliary body involvement, older age, epithelioid subtype and large tumor basal diameters are tumor features associated with poor prognosis [14]. Recent knowledge of detailed molecular mechanisms underlying UM has led to more accurate prognostic predictions. The $8 q$ gain mutation has been associated with increased risk of metastasis while monosomy 3 has been associated with a decreased risk [15]. Additionally, gene expression profiling has proven to be a superior predictor of prognostic and metastatic potential in UM. Finally, detection of circulating tumor DNA is another important predictive factor for developing metastasis [16].

Given this constantly evolving treatment paradigm, herein, we evaluate the published data on local therapeutic options for non-metastatic UM and propose a functional treatment algorithm (Fig. 1).

\section{Treatment Modalities}

Most common treatment options for non-metastatic UM include surgery, plaque brachytherapy, and/or particle beam radiotherapy (RT). Surgical options include local resection, enucleation and orbital exenteration. Local resection can be achieved either by exoresection, which involves en bloc tumor removal via a scleral approach, or by endoresection, which is piecemeal removal via a vitreoretinal approach. These eye-conserving treatment approaches, in recent years, are now preferred over enucleation [17]. Enucleation is the surgical removal of the eye itself sparing the extraocular muscles and remaining orbital contents. Orbital exenteration involves the surgical removal of the entire orbital tissue including the eye, periorbita, appendages and eyelids. It is the preferred surgical approach for patients with large extraocular invasion or orbital extension. One added benefit of a surgical therapeutic ap- 


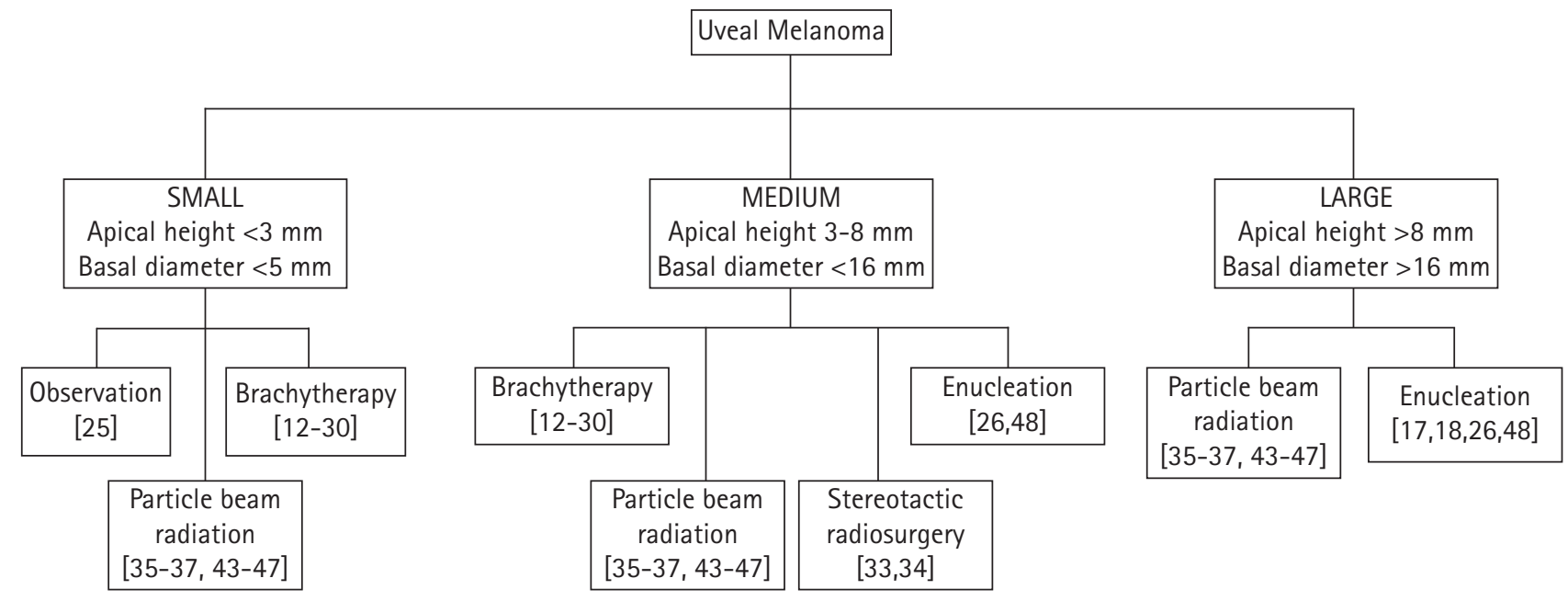

Fig. 1. Treatment algorithm for the management of nonmetastatic uveal melanoma.

proach is the ability to obtain adequate tissue samples for a detailed histopathologic and genetic analyses.

Plaque brachytherapy or plaque radiotherapy is the most widely used treatment modality in the management of UM and involves administration of a fixed dose of RT to the tumor. This is achieved by insertion of a radioactive implant into the episcleral tissue that delivers an apex RT dose of 80-100 Gy $[18,19]$. The most frequently employed radioisotope in the treatment of UM is lodine-125 $\left({ }^{125} \mathrm{I}\right)$ owing to its favorable dosimetric profile, followed by Ruthenium-106 $\left({ }^{106} \mathrm{Ru}\right)$, and Palladium-103 $\left({ }^{103} \mathrm{Pd}\right)$ [20]. The most common complications of brachytherapy administration are retinopathy, cataract formations, macular edema, neovascular glaucoma, dry eye, keratitis, eye pain, and scleral necrosis. Finally, improved early outcomes have been noted in UM patients when brachytherapy administration has been aided with the use of additional techniques such as intraoperative ultrasound guidance and echographic confirmation of plaque placement [21].

Particle beam therapy (PBT) or charged-particle radiotherapy (CPRT) is the second most frequently used form of RT in the treatment of UM. Protons, helium ions and carbon ions are delivered as highly precise external RT beams with a pre- specified dose. When PBT is utilized, a RT dose of 50-70 cobalt gray equivalent (CGyE) is usually delivered in 4 to 5 fractions. When carbon ions are used, a dose of 60-85 CGyE is delivered in 4 to 5 fractions. Owing to their physical properties, charged particles provide increased targeting, especially at the end of the beam range [22]. Furthermore, the usage of tantalum chips and volumetric planning in three dimensions also lead to optimal dosage administration. However, in spite of a precise homogenous dose delivery to the tumor, CPRT can also cause damage to the surrounding normal ocular structures leading to toxicities such as maculopathy, retinal detachment, glaucoma, cataract, vitreous hemorrhage and papillopathy [23]

\section{Current Treatment Strategies based on Size of UM Tumor}

The overall tumor size for UM is assessed based on both the apical height as well as the largest basal diameter of the tumor and is classified based on guidelines from the Collaborative Ocular Melanoma Study (COMS) group [24].

\section{Small tumors}

A landmark COMS study elucidated that tumors measuring $<3$ $\mathrm{mm}$ in apical height along with having a basal diameter measuring $<5 \mathrm{~mm}$ should be primarily managed with observation [24]. There was no difference seen between patients enrolled in the study who received immediate therapeutic intervention versus those who pursued close observation and therefore the conclusion was to reserve treatment only at the time of tumor growth. Only $21 \%$ of patients on this trial demonstrated tumor growth in 2 years while 31\% had tumor growth at 5 years post diagnosis.

\section{Medium tumors}

Medium tumors are defined as an apical height of 3-8 $\mathrm{mm}$ and a basal diameter of $<16 \mathrm{~mm}$. Treatment options for these patients range from plaque brachytherapy to PBT to enucleation. Another landmark COMS study, conducted over a span of 10 years, evaluated the quality of life after ${ }^{125}$ I plaque brachytherapy (IBT) or enucleation in 209 patients with choroidal melanoma. They concluded that there was no significant difference in survival between the two groups, but revealed that there was better visual function, defined as peripheral vision, for up to 2 years after treatment in pa- 
Table 1. Studies of plaque brachytherapy in uveal melanoma

\begin{tabular}{|c|c|c|c|c|c|c|}
\hline Study & Radioisotope & $n$ & Tumor thickness ${ }^{\mathrm{a})}(\mathrm{mm})$ & Tumor diameter ${ }^{\mathrm{a})}(\mathrm{mm})$ & Follow-up ${ }^{\text {a) }}(y r)$ & Local control (\%) \\
\hline Shields et al. [27] & ${ }^{125} \mathrm{I}(\mathrm{TTT})$ & 272 & 4.0 & 11.0 & 5 & 97.0 \\
\hline Verschueren et al. [30] & ${ }^{106} \mathrm{Ru}$ & 425 & 4.2 & 10.9 & 5 & 96.0 \\
\hline Takiar et al. [28] & ${ }^{106} \mathrm{Ru}$ & 40 & 3.1 & 9.6 & 5 & 97.0 \\
\hline Tarmann et al. [29] & ${ }^{106} \mathrm{Ru}$ & 143 & 4.5 & 11.0 & 4 & 85.3 \\
\hline Finger et al. [26] & ${ }^{103} \mathrm{Pd}$ & 400 & $\mathrm{~N} / \mathrm{A}$ & NA & 4.2 & 96.7 \\
\hline
\end{tabular}

I, iodine; Ru, ruthenium; Pd, palladium; TI, transpupillary thermotherapy; NA, not available.

${ }^{\text {a) Mean values. }}$

tients who underwent IBT when compared to enucleation [25].

Several other studies have been conducted with an aim to explore the use of different radioisotopes in plaque brachytherapy for UM [26-30] (Table 1). Verschueren et al. [30], analyzed the longterm outcomes of ${ }^{106} \mathrm{Ru}$ brachytherapy in 425 patients with small or intermediate UM. They observed a 5-year local control (LC) and overall control of $96 \%$ and $79.6 \%$, respectively and also revealed functional and cosmetic eye preservation rates at 5 years of $52 \%$ and 96\%, respectively. Takiar et al. [28] also demonstrated excellent tumor control and acceptable toxicity levels after ${ }^{106} \mathrm{Ru}$ brachytherapy in a cohort study of 40 patients with UM. Actuarial 5-year LC and overall survival were 97\% and 92\%, respectively. Enucleation was not required in any of the patients and there was no diagnosis of neovascular glaucoma at follow-up. Tarmann et al. [29] evaluated the medical records of 143 patients managed with ${ }^{106} \mathrm{Ru}$ brachytherapy for UM and demonstrated excellent rates for tumor control with a 2- and 4-year recurrence rate of $8.4 \%$ and $14.7 \%$, respectively. They also revealed promising eye-preservation results with the likelihood of keeping the eye in $94.7 \%$ of the patients at 24 months and $91.8 \%$ at 48 months post-brachytherapy. ${ }^{103} \mathrm{Pd}$ plaque brachytherapy was evaluated by Finger et al. [26] in a retrospective case series of 400 patients with UM. They concluded that ${ }^{103} \mathrm{Pd}$ provided a superior option compared to alternative forms of radiation and demonstrated a local control of $96.7 \%$; only 14 patients in the study required enucleation at a later date. Larger trials are needed to ascertain the optimal dosage of IBT in the treatment of UM.

Finally, more recent data is emerging, looking at additional treatment options in conjunction with IBT. Use of intravitreal bevacizumab at the time of plaque removal and at 4-month intervals for a period of 2 years in 292 patients with UM showed significantly decreased macular edema and vision loss in these patients [31]. Additionally a prospective non-comparative interventional case series by Shields et al. [27] in 270 patients with choroidal melanoma studied the effectiveness of IBT followed by transpupillary thermotherapy (TT). $\Pi$ Tा is a non-invasive treatment option where infrared lasers are delivered to the tumor and is mostly effective for smaller low-risk tumors [32]. Shields et al. [27] demonstrated that plaque brachytherapy followed by 3 sessions of $\Pi \pi$ resulted in a tumor recurrence of only $2 \%$ at 2-year follow-up and 3\% at 5-year follow-up.

Stereotactic photon beam radiosurgery (SRS) is another option that can be employed in the treatment of UM in the medium-sized tumor group. Gamma knife, CyberKnife or linear accelerator platforms are some of modalities utilized [33]. Sikuade et al. [34] conducted a review of 191 patients with UM who were managed with either SRS ( $n=85)$ or PBT $(n=106)$. They concluded that both treatments had excellent $\mathrm{LC}$ rates and eye preservation rates (98\% and $95 \%$ of SRS and PBT groups, respectively), but there was superior visual prognosis in the PBT group when compared to SRS (65\% vs. $45 \% ; p=0.008)$.

\section{Large tumors}

Several studies have been performed to compare the effectiveness of surgical procedures to brachytherapy in the management of large UM, defined as an apical height $>8 \mathrm{~mm}$ or a basal diameter of $>16 \mathrm{~mm}$ [35-38] (Table 2). The most notable therapies utilized are CPRT and enucleation. That being said, IBT is also considered a potential option for large tumors. A large retrospective, comparative, non-randomized study of 237 patients with large UM (defined as thickness $>7.5 \mathrm{~mm}$ ) by Bechrakis et al. [35], compared IBT to transscleral tumor resection (TSR) and demonstrated better visual acuity was retained in the TSR group (61.1\% vs. 5.6\%; $p<0.0009$ ) as well as lower incidence of secondary glaucoma in TSR group when compared to IBT (5.6\% vs. $33.3 \%$; $p=0.03$ ). There was no difference, however, in the mortality rates between the two groups. A matched case-control study by Kivela et al. [37], compared the complication rates, tumor control and visual acuity following IBT to TSR in 49 pairs of patients with large choroidal melanomas (thickness $>6 \mathrm{~mm}$ ). The results of this study suggested that the risk of losing 20/200 vision was higher after IBT compared to TSR (hazard ratio $[H R]=2.38 ; 95 \%$ confidence interval $[\mathrm{Cl}], 1.46-3.83 ; p<$ 0.001 ) but there was a lower risk of tumor recurrence after IBT compared to TSR $(\mathrm{HR}=0.02 ; 95 \% \mathrm{Cl}, 0.01-0.11 ; \mathrm{p}<0.001)$. $\mathrm{A}$ 
Table 2. Studies of TSR vs. IBT in uveal melanoma

\begin{tabular}{|c|c|c|c|c|c|}
\hline Study & $\mathrm{n}$ & $\begin{array}{l}\text { Tumor } \\
\text { height } \\
\text { (mm) }\end{array}$ & $\begin{array}{l}\text { Tumor } \\
\text { diameter } \\
(\mathrm{mm})\end{array}$ & VA in TSR and IBT & Risk of LR in TSR and IBT \\
\hline Bechrakis et al. [35] & 237 & 9.4 & 14.5 & $\begin{array}{c}\text { VA }>2 / 200 \text { in } 61.1 \% \text { TSR vs. 5.6\% IBT } \\
\qquad(p<0.0009)\end{array}$ & NA \\
\hline Kivela et al. [37] & 98 & 7.9 & NA & $\begin{array}{c}\mathrm{VA}<20 / 200 \text { after IBT }(\mathrm{HR}=2.38 ; 95 \% \mathrm{Cl}, \\
1.48-3.83 ; \mathrm{p}<0.001)\end{array}$ & $\begin{array}{c}\text { LR in IBT vs. TSR }(H R=0.02 ; 95 \% \mathrm{Cl}, 0.01-0.11 ; \\
p<0.001)\end{array}$ \\
\hline Caminal et al. [36] & 72 & 10.0 & 15.0 & $\begin{array}{c}\text { VA }<20 / 200 \text { in } 46.7 \% \text { TSR vs. } 68.8 \% \text { IBT } \\
(p<0.121)\end{array}$ & LR in TSR $10.5 \%$ vs. IBT 5.7\% $(p<0.602)$ \\
\hline Puusaari et al. [38] & 87 & 10.8 & 13.3 & $\begin{array}{c}\text { VA }<20 / 400 \text { in } 60 \% \text { TSR }(95 \% \mathrm{Cl}, 35-75) \text { vs. } \\
75 \% \text { IBT }\left(95 \% \mathrm{Cl}^{2} 59-86\right)\end{array}$ & $\begin{array}{c}5 \text {-year LR } 41 \% \text { in TSR }(95 \% \mathrm{Cl}, 17-63) \text { vs. } 7 \% \text { in } \\
\text { IBT }(95 \% \mathrm{Cl}, 2-17)\end{array}$ \\
\hline
\end{tabular}

TSR, transscleral resection; IBT, lodine-125 brachytherapy; VA, visual acuity; LR, local recurrence; HR, hazard ratio; $\mathrm{Cl}$, confidence interval; NA, not available.

${ }^{a)}$ Mean values.

retrospective study by Puusaari et al. [38], in 87 patients with large UM compared TSR to IBT revealed promising results for improving visual acuity in the TSR group but also noted an increase in rates of local recurrence. They observed that the 2-year incidence of losing $20 / 400$ vision was 60\% (95\% Cl, 35-75) for TSR group and 75\% (95\% Cl, 59-86) for IBT group but the risk of 5-year local recurrence in TSR group and IBT group was 41\% (95\% Cl, 17-63) and $7 \%$ (95\% Cl, 2-17), respectively. The Zimmerman-McLean-Foster hypothesis suggests that rates of tumor recurrence is higher after surgical intervention of UM due to tumor manipulation during the procedures which may accelerate tumor cell dissemination $[39,40]$. Despite this hypothesis, given improved visual acuity and equivalent survivals, TSR has been advocated as an alternative to enucleation and RT in the treatment of large uveal melanomas [41,42].

Regarding CPRT, there are many studies detailing the use of PBT in comparison with other treatment modalities for UM [34,43-46] (Table 3). Abrams et al. [47] conducted a survival analysis of 1,004 cases of UM in which 380 cases were managed with external beam radiotherapy (EBRT) and 624 cases were managed with plaque brachytherapy. No difference in 5-year overall survival was seen between the two groups (83.3\% EBRT vs. 82.5\% BT; $p=0.69$ ). Caujolle et al. [44] performed a retrospective study in 886 patients with UM who were managed with PBT They observed LC rates of $93.9 \%$ and $92.1 \%$ at 5 and 10 years, respectively and also noted ocular conservation rates of $91.1 \%$ and $87.3 \%$ at 5 and 10 years, respectively. A prospective, interventional, noncomparative study performed in 2645 patients by Egger et al. [45], analyzed eye preservations rates in patients managed with PBT and found that overall eye retention rates were $88.9 \%, 86.2 \%$, and $83.7 \%$ at 5,10 , and 15 years, respectively. They concluded that favorable results were noted even for larger tumors and tumors near the optic disc. A retrospective, consecutive cohort study in 492 patients with large UM by Bensoussan et al. [43], noted good LC with overall and specific survival rates at 5 years of $65 \%$ and $75 \%$, respectively. They con-
Table 3. Studies of external beam radiotherapy in uveal melanoma

\begin{tabular}{lcccc}
\hline Study & Radiation & $\mathrm{n}$ & $\begin{array}{c}\text { Mean } \\
\text { follow-up (yr) }\end{array}$ & $\begin{array}{c}\text { Local control } \\
(\%)\end{array}$ \\
\hline Mishra et al. [46] & Helium ions & 86 & 14.6 & $100(5 \mathrm{yr})$ \\
& $\begin{array}{c}125 \text { I plaque } \\
\text { Sikuade et al. [34] }\end{array}$ & 98 & 12.3 & $84(5 \mathrm{yr})$ \\
& Protons & 106 & 2.8 & 97 \\
Caujolle et al. [44] & SRS & 85 & 3.3 & 100 \\
Egger et al. [45] & Protons & 886 & 5.3 & $94(5 \mathrm{yr})$ \\
Bensoussan et al. [43] & Protons & 2,645 & 3.6 & $99(5 \mathrm{yr})$ \\
\hline
\end{tabular}

I, iodine; SRS, stereotactic radiosurgery.

cluded that PBT can serve as an effective alternative to enucleation in patients with large tumors. The UCSF-LBNL randomized trial by Mishra et al. [46] conducted in 184 patients with UM, included 86 patients receiving Helium ion particle therapy and 98 patients receiving IBT. They noted significantly improved LC in particle arm compared to the IBT arm (100\% vs. $84 \%$ at 5 years, $98 \%$ vs. $79 \%$ at 12 years; $\log -r a n k p=0.0006)$. Significantly lower need for further enucleation was also demonstrated in particle arm (11\% vs. $22 \%$ at 5 years, $17 \%$ vs. $37 \%$ at 12 years; log-rank $p=0.01$ ). Given numerous prior studies revealed promising results, CPRT is now used as a definitive treatment option in large tumors. Furthermore, CPRT gains an obvious advantage when the UM is in a circumpapillary location surrounding the optic nerve, as it is not feasible to place a plaque completely around the tumor and hence CPRT is preferred. Additionally, in large tumors where IBT is not appropriate, CPRT is the preferred treatment modality over enucleation when eye preservation is desired [48].

Finally, Bechrakis and Foerster [49] developed a novel approach of combining neoadjuvant PBT and subsequent endoresection in 58 patients with large UM (thickness $>7 \mathrm{~mm}$ ). They concluded that high-risk patients did not have increased morbidity and showed a lower rate of ocular side effects such as cataracts and retinal detachment in short-term follow-up compared to patients with UM 
of smaller size (thickness $<7 \mathrm{~mm}$ ). A retrospective interventional case series by Willerding et al. [50], also studied the benefits of neoadjuvant PBT prior to TSR in 106 patients with UM. Local recurrence was noted in 5 patients while enucleation was required in 10 patients. The study concluded that there were no significant risk factors noted for local recurrence but also stated that additional vitreoretinal surgery was frequently needed (69.8\%).

In summary, surgical intervention such as enucleation is the preferred approach in large tumors which cannot be effectively managed with RT, especially if they are well-circumscribed or juxtapapillary in location. The use of neoadjuvant RT prior to performing surgery has been shown to produce improved results and a decrease in the potential risk of tumor seeding. Further evaluation of this combined modality approach is required but may be appropriate patients presenting with neovascular glaucoma, tumor replacing more than half of the globe, orbital invasion or optic nerve involvement.

\section{Conclusion}

UM, the most common primary intraocular malignancy, continues to provide daunting challenges in its treatment management. With more than half the patients developing metastatic disease after initial non-metastatic presentation, prompt diagnosis and treatment play a crucial role in alleviating the morbidity and mortality of this disease. Currently, RT is the most common treatment approach in the management of UM, especially for small and intermediate-sized tumors. IBT is the most frequently employed type of RT, followed by CPRT. The most common surgical approaches remain enucleation and local resection. In recent years, enucleation is considered as an option only in patients with large tumors or in those with optic nerve involvement where RT does not result in a favorable outcome. The popularity of local resection has also diminished of late, as RT provides a superior alternative; however, when local resection is performed as a primary treatment modality, it is often coupled with neoadjuvant or adjuvant RT to further decrease chances of local recurrence. Additional clinical trials and targeted therapies aimed at the molecular pathogenesis of UM may offer novel avenues in managing this disease in the future.

\section{Conflict of Interest}

No potential conflict of interest relevant to this article was reported.

\section{References}

1. Krantz BA, Dave N, Komatsubara KM, Marr BP, Carvajal RD. Uveal melanoma: epidemiology, etiology, and treatment of primary disease. Clin Ophthalmol 2017;11:279-89.

2. McLaughlin CC, Wu XC, Jemal A, Martin HJ, Roche LM, Chen VW. Incidence of noncutaneous melanomas in the U.S. Cancer 2005;103:1000-7.

3. Park SJ, Oh CM, Kim BW, Woo SJ, Cho H, Park KH. Nationwide incidence of ocular melanoma in South Korea by using the National Cancer Registry Database (1999-2011). Invest Ophthalmol Vis Sci 2015;56:4719-24.

4. Weis E, Shah CP, Lajous M, Shields JA, Shields CL. The association between host susceptibility factors and uveal melanoma: a meta-analysis. Arch Ophthalmol 2006;124:54-60.

5. Johansson $P$, Aoude $L G$, Wadt $K$, et al. Deep sequencing of uveal melanoma identifies a recurrent mutation in PLCB4. Oncotarget 2016;7:4624-31.

6. Schmidt-Pokrzywniak A, Jockel KH, Bornfeld N, Sauerwein W, Stang A. Positive interaction between light iris color and ultraviolet radiation in relation to the risk of uveal melanoma: a case-control study. Ophthalmology 2009;116:340-8.

7. de Lange MJ, Razzaq L, Versluis M, et al. Distribution of GNAO and GNA11 mutation signatures in uveal melanoma points to a light dependent mutation mechanism. PLoS One 2015;10:e0138002.

8. Pandiani C, Beranger GE, Leclerc J, Ballotti R, Bertolotto C. Focus on cutaneous and uveal melanoma specificities. Genes Dev 2017:31:724-43.

9. Mallet JD, Gendron SP, Drigeard Desgarnier MC, Rochette PJ. Implication of ultraviolet light in the etiology of uveal melanoma: a review. Photochem Photobiol 2014;90:15-21.

10. Damato EM, Damato BE. Detection and time to treatment of uveal melanoma in the United Kingdom: an evaluation of 2,384 patients. Ophthalmology 2012;119:1582-9.

11. Shields CL, Furuta M, Berman EL, et al. Choroidal nevus transformation into melanoma: analysis of 2514 consecutive cases. Arch Ophthalmol 2009;127:981-7.

12. Giuliari GP, McGowan HD, Pavlin CJ, Heathcote JG, Simpson ER. Ultrasound biomicroscopic imaging of iris melanoma: a clinicopathologic study. Am J Ophthalmol 2011;151:579-85.

13. Skinner CC, Augsburger JJ, Augsburger BD, Correa ZM. Comparison of alternative tumor size classifications for posterior uveal melanomas. Invest Ophthalmol Vis Sci 2017;58:3335-42.

14. Kuk D, Shoushtari AN, Barker CA, et al. Prognosis of mucosal, uveal, acral, nonacral cutaneous, and unknown primary melanoma from the time of first metastasis. Oncologist 2016;21:84854.

15. Versluis $\mathrm{M}$, de Lange MJ, van Pelt SI, et al. Digital PCR validates $8 \mathrm{q}$ dosage as prognostic tool in uveal melanoma. PLoS One 
2015;10:e0116371.

16. Bidard FC, Madic J, Mariani P, et al. Detection rate and prognostic value of circulating tumor cells and circulating tumor DNA in metastatic uveal melanoma. Int J Cancer 2014;134:1207-13.

17. Damato B. The role of eyewall resection in uveal melanoma management. Int Ophthalmol Clin 2006;46:81-93.

18. Damato B. Progress in the management of patients with uveal melanoma. The 2012 Ashton Lecture. Eye (Lond) 2012;26:115772.

19. Reichstein D, Karan K. Plaque brachytherapy for posterior uveal melanoma in 2018: improved techniques and expanded indications. Curr Opin Ophthalmol 2018;29:191-8.

20. Echegaray JJ, Bechrakis NE, Singh N, Bellerive C, Singh AD. Iodine-125 brachytherapy for uveal melanoma: a systematic review of radiation dose. Ocul Oncol Pathol 2017;3:193-8.

21. Aziz HA, Al Zahrani YA, Bena J, et al. Episcleral brachytherapy of uveal melanoma: role of intraoperative echographic confirmation. Br J Ophthalmol 2017;101:747-51.

22. Hocht $S$, Stark R, Seiler F, et al. Proton or stereotactic photon irradiation for posterior uveal melanoma? A planning intercomparison. Strahlenther Onkol 2005;181:783-8.

23. Lane AM, Kim IK, Gragoudas ES. Long-term risk of melanoma-related mortality for patients with uveal melanoma treated with proton beam therapy. JAMA Ophthalmol 2015;133:792-6.

24. Factors predictive of growth and treatment of small choroidal melanoma: COMS Report No. 5. The Collaborative Ocular Melanoma Study Group. Arch Ophthalmol 1997;115:1537-44.

25. Melia M, Moy CS, Reynolds SM, et al. Quality of life after iodine 125 brachytherapy vs enucleation for choroidal melanoma: 5-year results from the Collaborative Ocular Melanoma Study. COMS OOLS Report No. 3. Arch Ophthalmol 2006;124:226-38.

26. Finger PT, Chin KJ, Duvall G; Palladium-103 for Choroidal Melanoma Study Group. Palladium-103 ophthalmic plaque radiation therapy for choroidal melanoma: 400 treated patients. Ophthalmology 2009;116:790-6.

27. Shields CL, Cater J, Shields JA, et al. Combined plaque radiotherapy and transpupillary thermotherapy for choroidal melanoma: tumor control and treatment complications in 270 consecutive patients. Arch Ophthalmol 2002;120:933-40.

28. Takiar V, Gombos DS, Mourtada F, et al. Disease control and toxicity outcomes using ruthenium eye plaque brachytherapy in the treatment of uveal melanoma. Pract Radiat Oncol 2014;4:e18994.

29. Tarmann L, Wackernagel W, Avian A, et al. Ruthenium-106 plaque brachytherapy for uveal melanoma. Br J Ophthalmol 2015;99:1644-9.

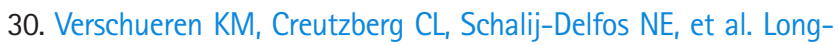

term outcomes of eye-conserving treatment with Ruthenium(106) brachytherapy for choroidal melanoma. Radiother Oncol 2010;95:332-8.

31. Shah SU, Shields CL, Bianciotto CG, et al. Intravitreal bevacizum$\mathrm{ab}$ at 4-month intervals for prevention of macular edema after plaque radiotherapy of uveal melanoma. Ophthalmology 2014;121:269-75.

32. Mashayekhi A, Shields $C L$, Rishi $P$, et al. Primary transpupillary thermotherapy for choroidal melanoma in 391 cases: importance of risk factors in tumor control. Ophthalmology 2015;122:600-9.

33. Zytkovicz A, Daftari I, Phillips TL, Chuang CF, Verhey L, Petti PL. Peripheral dose in ocular treatments with CyberKnife and Gamma Knife radiosurgery compared to proton radiotherapy. Phys Med Biol 2007;52:5957-71.

34. Sikuade MJ, Salvi S, Rundle PA, Errington DG, Kacperek A, Rennie IG. Outcomes of treatment with stereotactic radiosurgery or proton beam therapy for choroidal melanoma. Eye (Lond) 2015;29:1194-8.

35. Bechrakis NE, Bornfeld N, Zoller I, Foerster MH. lodine 125 plaque brachytherapy versus transscleral tumor resection in the treatment of large uveal melanomas. Ophthalmology 2002;109:185561.

36. Caminal JM, Padron-Perez N, Arias L, et al. Transscleral resection without hypotensive anaesthesia vs iodine-125 plaque brachytherapy in the treatment of choroidal melanoma. Eye (Lond) 2016;30:833-42.

37. Kivela T, Puusaari I, Damato B. Transscleral resection versus iodine brachytherapy for choroidal malignant melanomas 6 millimeters or more in thickness: a matched case-control study. Ophthalmology 2003;110:2235-44.

38. Puusaari I, Damato B, Kivela T. Transscleral local resection versus iodine brachytherapy for uveal melanomas that are large because of tumour height. Graefes Arch Clin Exp Ophthalmol 2007;245:522-33.

39. Singh AD, Rennie IG, Kivela T, Seregard S, Grossniklaus H. The Zimmerman-McLean-Foster hypothesis: 25 years later. Br J Ophthalmol 2004;88:962-7.

40. Zimmerman LE, McLean IW, Foster WD. Does enucleation of the eye containing a malignant melanoma prevent or accelerate the dissemination of tumour cells. Br J Ophthalmol 1978;62:420-5.

41. Bechrakis NE, Petousis V, Willerding $G$, et al. Ten-year results of transscleral resection of large uveal melanomas: local tumour control and metastatic rate. Br J Ophthalmol 2010;94:460-6.

42. Konstantinidis L, Groenewald C, Coupland SE, Damato B. Longterm outcome of primary endoresection of choroidal melanoma. $\mathrm{Br} J$ Ophthalmol 2014;98:82-5.

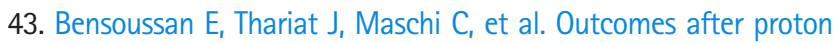


beam therapy for large choroidal melanomas in 492 patients. Am J Ophthalmol 2016;165:78-87.

44. Caujolle JP, Mammar H, Chamorey E, Pinon F, Herault J, Gastaud P. Proton beam radiotherapy for uveal melanomas at nice teaching hospital: 16 years' experience. Int J Radiat Oncol Biol Phys 2010;78:98-103.

45. Egger $E$, Zografos $L$, Schalenbourg $A$, et al. Eye retention after proton beam radiotherapy for uveal melanoma. Int J Radiat Oncol Biol Phys 2003;55:867-80.

46. Mishra KK, Quivey JM, Daftari IK, et al. Long-term results of the UCSF-LBNL randomized trial: charged particle with helium ion versus iodine-125 plaque therapy for choroidal and ciliary body melanoma. Int J Radiat Oncol Biol Phys 2015;92:376-83.
47. Abrams MJ, Gagne NL, Melhus CS, Mignano JE. Brachytherapy vs. external beam radiotherapy for choroidal melanoma: survival and patterns-of-care analyses. Brachytherapy 2016;15:216-23.

48. Gragoudas ES. Proton beam irradiation of uveal melanomas: the first 30 years. The Weisenfeld Lecture. Invest Ophthalmol Vis Sci 2006;47:4666-73.

49. Bechrakis NE, Foerster MH. Neoadjuvant proton beam radiotherapy combined with subsequent endoresection of choroidal melanomas. Int Ophthalmol Clin 2006;46:95-107.

50. Willerding GD, Cordini D, Moser L, Krause L, Foerster MH, Bechrakis NE. Neoadjuvant proton beam irradiation followed by transscleral resection of uveal melanoma in 106 cases. Br J Ophthalmol 2016;100:463-7. 